\title{
High-quality GaN films grown on chemical vapor-deposited graphene films
}

\author{
Kunook Chung ${ }^{1}$, Suk In Park ${ }^{1}$, Hyeonjun Baek ${ }^{1}$, Jin-Seok Chung ${ }^{2}$ and Gyu-Chul Yi ${ }^{1}$ \\ We report the growth of high-quality GaN films on large-size graphene films for visible light-emitting diodes (LEDs). \\ The graphene films were synthesized by chemical vapor deposition and then transferred onto amorphous silica $\left(\mathrm{SiO}_{2}\right)$ substrates \\ that do not have an epitaxial relationship with GaN. Before growing the high-quality GaN thin films, $\mathrm{ZnO}$ nanowalls were grown \\ on the graphene films as an intermediate layer. The structural and optical characteristics of the GaN films were investigated, \\ and the films exhibited stimulated emission even at room temperature, a highly $c$-axis-oriented crystal structure, and a preferred \\ in-plane orientation. Visible LEDs that emitted strong electroluminescence under room illumination were fabricated using the \\ GaN thin films. \\ NPG Asia Materials (2012) 4, e24; doi:10.1038/am.2012.45; published online 7 September 2012
}

Keywords: amorphous substrate; gallium nitride; hybrid heterostructure; large-size graphene film; light-emitting diode

\section{INTRODUCTION}

Hybrid heterostructures composed of high-quality inorganic thin films grown directly on atomic-scale layered sheets, such as graphene and boron nitride, offer a novel material system for transferable electronics and optoelectronics. ${ }^{1-3}$ Although graphene has been considered to be an ideal supporting layer for the growth of inorganic materials because of its high thermal and mechanical stability and flexibility, inorganic semiconductor thin films exhibit excellent material characteristics, including a high carrier mobility, a radiative recombination rate and long-term stability. For example, as recently reported, graphene layers exfoliated mechanically from graphite powder and their hybrid heterostructures with inorganic semiconductor films are transferable onto arbitrary substrates, such as glass, metal or plastic. ${ }^{1}$ Nevertheless, the size limitation associated with mechanically exfoliated graphene has been a major obstacle for using inorganic semiconductors grown on graphene for many device applications, including displays and solar cells. Recently, large-size graphene films have become available, and hybrid heterostructures using these large-scale graphene films open up significant opportunities to fabricate various electronic and optoelectronic devices for commercial use. Here, we describe a method of growing high-quality GaN films on large-scale chemical vapor-deposited (CVD) graphene films and fabricating light-emitting devices directly on the films prepared on amorphous substrates. Furthermore, the light-emitting diodes (LEDs) fabricated on the graphene-coated amorphous substrates exhibited strong light emission under room illumination.
Optoelectronic devices fabricated on large-size substrates are of significant interest for applications to general illumination light sources, flat panel displays and solar cells. ${ }^{4,5}$ For device applications, the growth of amorphous inorganic semiconductors ${ }^{6,7}$ and organic thin films ${ }^{8}$ on amorphous substrates has widely been studied. However, the devices based on single-crystalline compound semiconductors such as GaN show much higher efficiencies, reliability and long-term stability. ${ }^{9,10}$ Nevertheless, because highquality, single-crystalline nitride films could be grown only on a lattice-matched single-crystal substrate, the use of amorphous substrates, such as fused silica, yielded poor thin film quality, including a rough surface morphology and a polycrystalline structure. ${ }^{11}$ Thus, the obstacle to growing large high-quality nitride films on large-size amorphous substrates must be resolved in order to use inorganic LEDs in displays and flat light sources. ${ }^{12}$

\section{MATERIALS AND METHODS}

Growth of GaN thin films on CVD graphene films

Large CVD graphene films were used as a substrate for the growth of highquality GaN films and the fabrication of LEDs on amorphous $\mathrm{SiO}_{2}$ substrates. The graphene films were prepared on $\mathrm{Cu}$ foil by a previously reported method. ${ }^{13}$ The films exhibited excellent optical transparencies and had fairly uniform and homogenous surface morphologies. Then, the CVD graphene films were transferred onto amorphous $\mathrm{SiO}_{2}$ substrates. Before the transfer process, the $\mathrm{Cu}$ foil was removed from the graphene films. Then, an oxygen plasma treatment was performed to enhance the formation of $\mathrm{ZnO}$ nanowalls across the entire surface of the graphene films. The oxygen plasma was applied

\footnotetext{
${ }^{1}$ National Creative Research Initiative Center for Semiconductor Nanorods and Department of Physics and Astronomy, Seoul National University, Seoul, Korea and ${ }^{2}$ Department of Physics, Soongsil University, Seoul, Korea

Correspondence: Professor G-C Yi, National Creative Research Initiative Center for Semiconductor Nanorods and Department of Physics and Astronomy, Seoul National University, Seoul 151-747, Korea.

E-mail: gcyi@snu.ac.kr

Received 23 April 2012; revised 8 July 2012; accepted 17 July 2012
} 
to the CVD graphene films for $1 \mathrm{~s}$ at an oxygen pressure of $100 \mathrm{mTorr}$ and a plasma power of $30 \mathrm{~W}$.

Nitride thin films were grown on CVD graphene films using an intermediate layer of $\mathrm{ZnO}$ nanowalls. After preparing the oxygen plasma-treated graphene films, vertically aligned $\mathrm{ZnO}$ nanowalls were grown by metal-organic chemical vapor deposition (inset of Figure 1a). The height and density of the $\mathrm{ZnO}$ nanowalls were $200-400 \mathrm{~nm}$ and $10^{10} \mathrm{~cm}^{-2}$, respectively. GaN epitaxial layers were grown on the $\mathrm{ZnO}$-coated graphene films using three-step growth by metal-organic chemical vapor deposition. In the first step, a thin $\mathrm{GaN}$ protecting layer was grown at $540-600{ }^{\circ} \mathrm{C}$ for 10 min with nitrogen as an ambient gas to prevent the degradation of $\mathrm{ZnO}$ nanowalls and prohibit reactions between $\mathrm{ZnO}$ and $\mathrm{GaN}$ layers at a higher temperature. In the second step, $\mathrm{GaN}$ layers were grown at temperatures of $1000-1100{ }^{\circ} \mathrm{C}$ for 15 min with hydrogen as an ambient gas to improve the lateral overgrowth of GaN layers. Finally, after further growth for $1 \mathrm{~h}$ at $1120-1180^{\circ} \mathrm{C}$, high-quality GaN layers were obtained.

\section{Measurements}

The morphology of GaN thin films grown on graphene-coated amorphous $\mathrm{SiO}_{2}$ substrates was investigated by scanning electron microscopy (JEOL 6510, Tokyo, Japan), and the crystal structure was determined by an X-ray
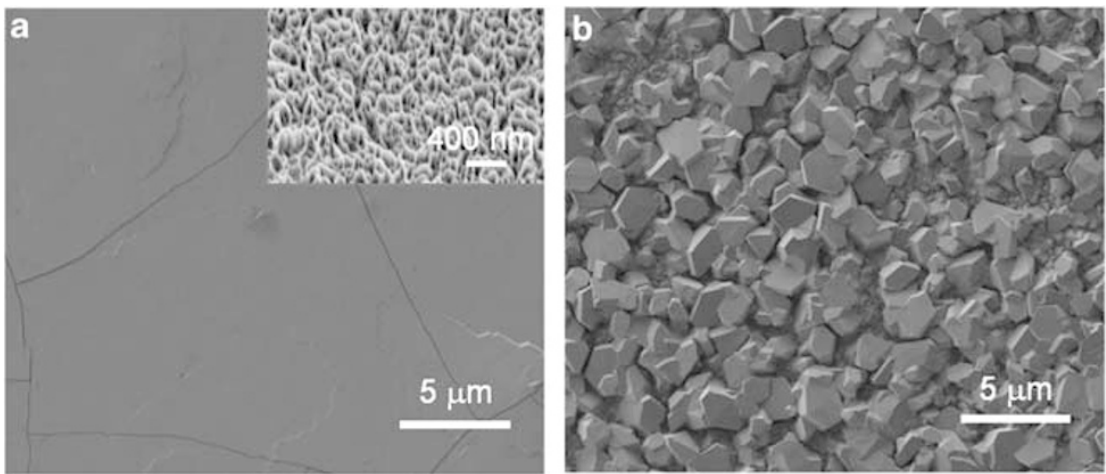

Figure 1 Effect of CVD graphene film as a substrate on the surface morphology of GaN films grown on amorphous substrates. Surface morphologies of GaN films grown on amorphous $\mathrm{SiO}_{2}$ substrates coated with CVD graphene films (a) and without CVD graphene films (b). The inset of a shows $\mathrm{ZnO}$ nanowalls grown on oxygen plasma-treated CVD graphene films at a tilting angle of $30^{\circ}$.
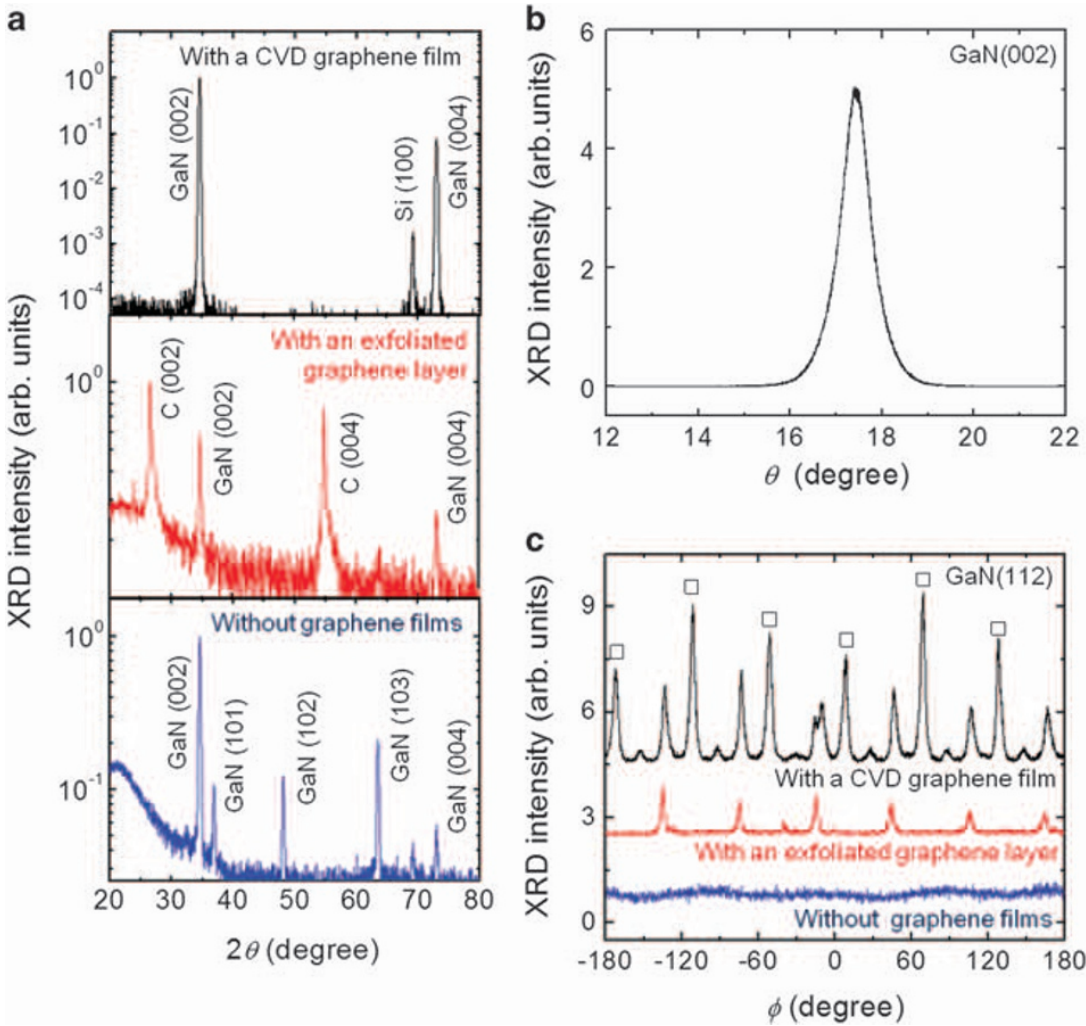

Figure 2 X-ray diffraction (XRD) spectra of GaN films grown on amorphous $\mathrm{SiO}_{2}$ substrates. (a) $\theta-2 \theta$ scans of GaN films made with CVD graphene films (top), with mechanically exfoliated graphene layers (middle), and without graphene films (bottom). (b) Rocking curve of GaN films on CVD graphene films. (c) $\phi$-scans of GaN films made with CVD graphene films (black solid line), with mechanically exfoliated graphene layers (red solid line), and without graphene films (blue solid line). 
diffractometer (PANalytical X'pert Pro, Almelo, Netherlands). The electroluminescence (EL) and current-voltage $(I-V)$ characteristics of the devices were measured by applying a DC voltage to the device using a source meter (Keithley 2400, Cleveland, OH, USA). The EL and photoluminescence (PL) spectra were measured using a detection system equipped with a monochromator and a charge-coupled device. A continuous He-Cd laser $(325 \mathrm{~nm})$ or a pulsed Nd:YAG laser $(355 \mathrm{~nm})$ was employed as an optical excitation source for the PL spectroscopy. The temperature-dependent PL measurements were performed using a He Displex refrigerating system.

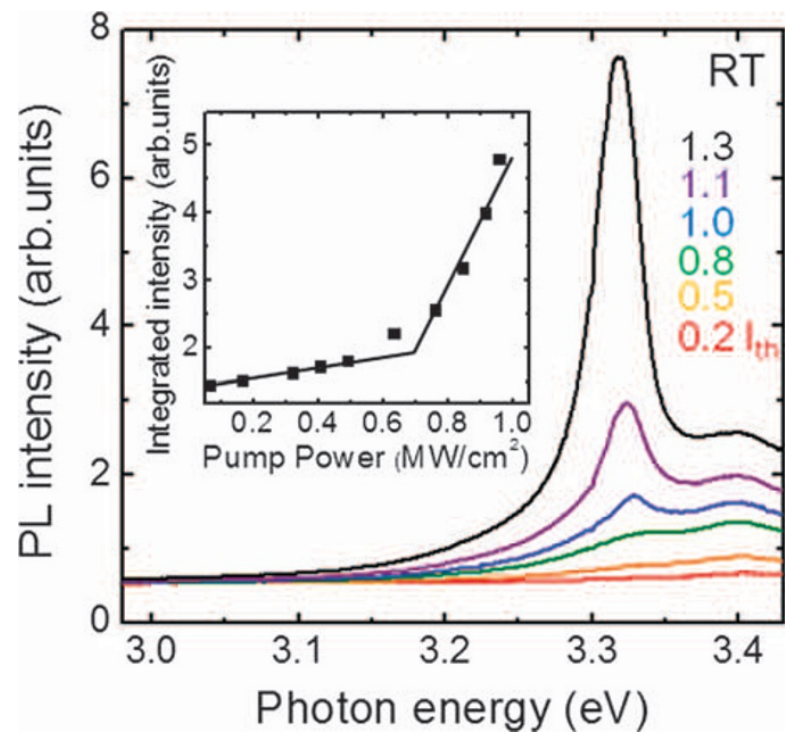

Figure 3 Power-dependent PL spectra of GaN films grown on CVD graphene film-coated $\mathrm{SiO}_{2}$ substrates at room temperature. The inset of the figure shows the integrated PL emission intensity as a function of the optical excitation.

\section{RESULTS AND DISCUSSION}

We investigated the effect of using a CVD graphene film as an intermediate layer on the surface morphology of GaN films grown on amorphous substrates. As shown in Figure 1a, GaN films grown on a CVD graphene-coated amorphous $\mathrm{SiO}_{2}$ substrate exhibited a flat and smooth morphology, presumably due to the heteroepitaxial relations between $\mathrm{GaN}$ on $\mathrm{ZnO}$ and $\mathrm{ZnO}$ on graphene. Meanwhile, a rough surface morphology with a few micron-sized irregular hillocks was observed for films directly grown on the $\mathrm{SiO}_{2}$ substrates in the absence of a graphene film (Figure $1 \mathrm{~b}$ ), indicating that the CVD graphene film significantly affects the surface morphology of the GaN film. In addition, the surface roughness was reduced using a CVD graphene film: the root mean square surface roughness over a $5 \times 5 \mu \mathrm{m}$ area was $11 \mathrm{~nm}$ for GaN films grown using the graphene film, 20 times smaller than that for GaN films grown directly on the amorphous substrates.

The crystal structure and growth orientation of the GaN films grown on CVD graphene films were examined by X-ray diffraction. Figure 2 a shows $\theta-2 \theta$ scan results for GaN films grown on various amorphous substrates using a CVD graphene film and mechanically exfoliated graphene as well as GaN films grown without using any graphene layers. Diffraction peaks for the GaN films grown on the substrates coated with graphene films were observed only at $34.47^{\circ}$ and $72.87^{\circ}$. These peaks correspond to the (002) and (004) orientations of wurtzite GaN, respectively. Additional (101), (102) and (103) diffraction peaks were observed for GaN films grown directly on amorphous substrates in the absence of graphene films. Because the GaN films were highly oriented in the $c$-axis direction, X-ray diffraction rocking curves could also be measured. For GaN films grown on the substrates with CVD graphene films, the full width at half maximum value of the X-ray diffraction rocking curves was as small as $0.8^{\circ}$ (Figure $2 \mathrm{~b}$ ), indicating the good $c$-axis orientation of the GaN film. This full width at half maximum value is much smaller than previously reported values $\left(3-6^{\circ}\right)$ for $\mathrm{GaN}$ films grown on

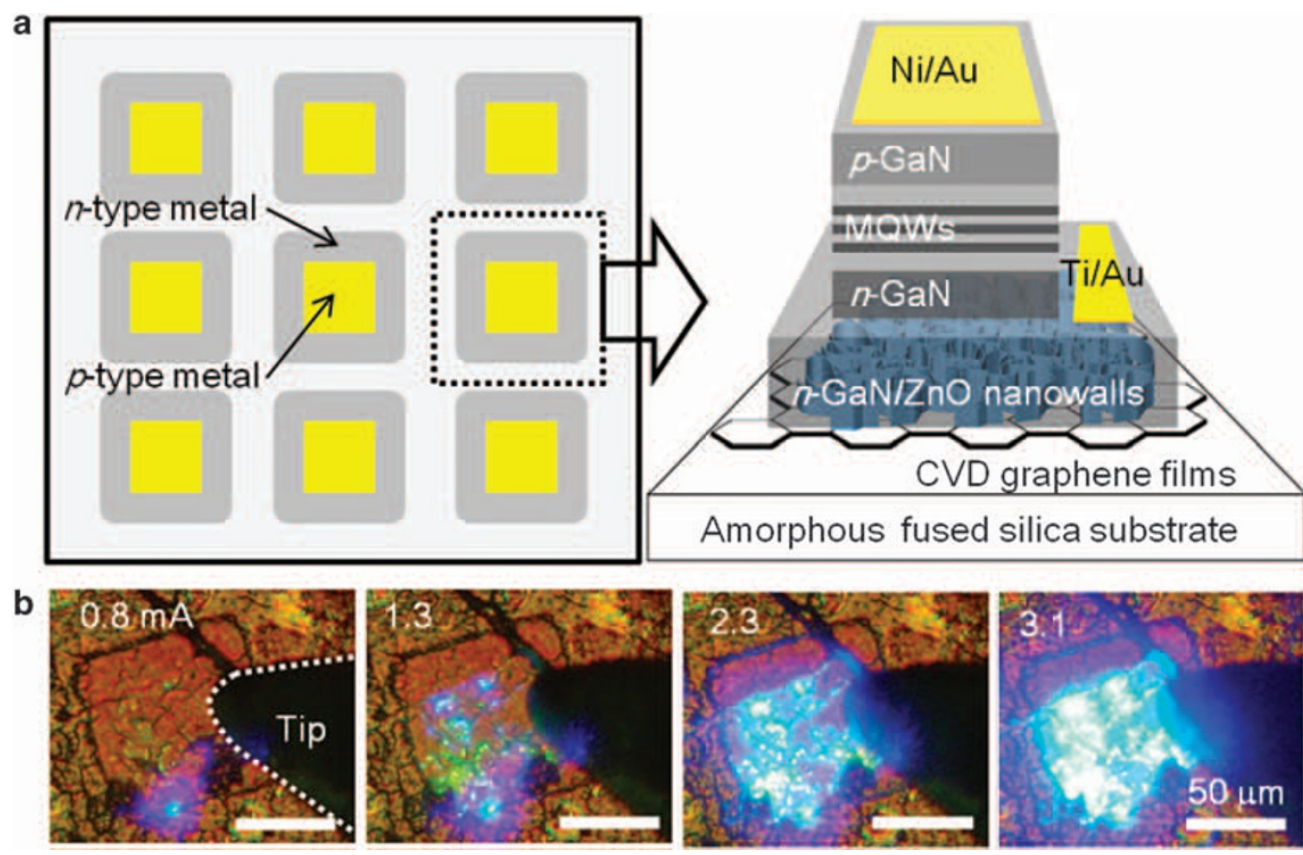

Figure 4 Visible thin-film LEDs fabricated on CVD graphene-coated amorphous $\mathrm{SiO}_{2}$ substrates. (a) Schematics of an array (left) of LED pixels and one LED pixel (right) that is composed of $n$-GaN, multiple quantum wells, and $p$-GaN layers. (b) EL emission images of the LEDs under room illumination at different applied currents. 
mechanically exfoliated graphene layers and on $\mathrm{SiO}_{2}$ substrates using a $\mathrm{ZnO}$ layer. ${ }^{14}$ This result strongly suggests that the graphene film has a critical role in forming GaN films with good vertical alignment.

We also investigated the in-plane orientation of the GaN films by measuring azimuthal $(\phi)$ scans of the $\{112\}$ diffraction. As shown in Figure 2c, whereas the $\phi$-scan (blue solid line) of the GaN film grown without a CVD graphene film showed no distinct peaks, three different peaks displaying $20^{\circ}$ shifts and sixfold symmetry (black solid line) were observed for the GaN films grown using a CVD graphene film. This result indicated that the GaN grains in the thin film had large grain angle boundaries and preferred in-plane orientations. Similar in-plane orientations for CVD graphene films synthesized on $\mathrm{Cu}$ foil have previously been reported, ${ }^{15-17}$ suggesting that the in-plane orientation of $\mathrm{GaN}$ results from using the CVD graphene film. Only one set of peaks possessing sixfold symmetry was observed for the $\phi$-scan (red solid line) of GaN films grown on an exfoliated graphene layer, indicating that the GaN films were single crystals without large-angle grain boundaries, similar to previous reports. ${ }^{18}$

The optical characteristics of GaN films grown on CVD graphenecoated amorphous $\mathrm{SiO}_{2}$ substrates were investigated using PL spectroscopy. Figure 3 shows the power-dependent PL spectra measured at room temperature using a pulsed Nd:YAG laser. At a low excitation laser power, below the threshold value $\left(I_{\text {th }}\right)$ of the optical pumping power, the GaN thin films exhibited a dominant PL peak at $3.40 \mathrm{eV}$ due to spontaneous emission. For excitation powers above the $I_{\mathrm{th}}$, the GaN films exhibited a strong and sharp PL peak at $3.33 \mathrm{eV}$ rising from the shoulder of the dominant peak. The integrated $\mathrm{PL}$ intensities of the GaN films increased superlinearly with the pumping power above the $I_{\text {th }}$, consistent with stimulated emission (inset of Figure 3). The $I_{\text {th }}$ of the GaN films grown on the graphenecoated amorphous substrates was $0.6-0.7 \mathrm{MW} \mathrm{cm}^{-2}$, comparable to previously reported values for $\mathrm{GaN}$ films grown on mechanically exfoliated graphene layers and on single-crystal sapphire and silicon substrates. ${ }^{1,19,20}$ The high optical quality of the GaN films was also confirmed as determined by low-temperature PL spectroscopy (see Supplementary Figure S1).

The GaN films grown on CVD graphene-coated amorphous $\mathrm{SiO}_{2}$ substrates could be used with many optoelectronic devices, such as LEDs and solar cells, because they are of high optical quality. We fabricated LEDs as an example of a device application. Although the nitride thin films grown on CVD graphene films are not single crystals, the flat and uniform surface morphology with a high $c$-axis orientation of the nitride thin films enables us to fabricate LEDs even on amorphous $\mathrm{SiO}_{2}$ substrates. The LEDs were composed of an $n$-GaN layer, three-period $\mathrm{In}_{x} \mathrm{Ga}_{1-x} \mathrm{~N} / \mathrm{GaN}$ multiple quantum wells (MQWs), and a $p$-GaN layer (Figure 4a). After growing the LED structures, lithography and $\mathrm{BCl}_{3}$ reactive ion etching were used for the exposure of $n$-GaN layers. $\mathrm{Ni} / \mathrm{Au}$ (with a thickness of $8 / 8 \mathrm{~nm}$ ) and $\mathrm{Ti} / \mathrm{Au}(20 / 20 \mathrm{~nm})$ bilayers were deposited on the $p$ - and $n$-GaN surfaces, respectively, and annealed to make ohmic contacts and reduce the contact resistance of the electrodes.

The GaN-based thin-film LEDs fabricated on CVD graphene-coated amorphous $\mathrm{SiO}_{2}$ substrates emitted strong blue light emissions that could be seen with the unaided eye under typical room illumination conditions (Figure $4 \mathrm{~b}$ ). The light emission from the LED at a driving current of $3.1 \mathrm{~mA}$ was fairly uniformly distributed over the $p$-contact area of $70 \times 70 \mu \mathrm{m}^{2}$. This result suggests that the hybrid heterostructure, composed of inorganic semiconductors directly grown on largescale graphene films, constitutes a new functional material system for developing unconventional inorganic optoelectronic devices in
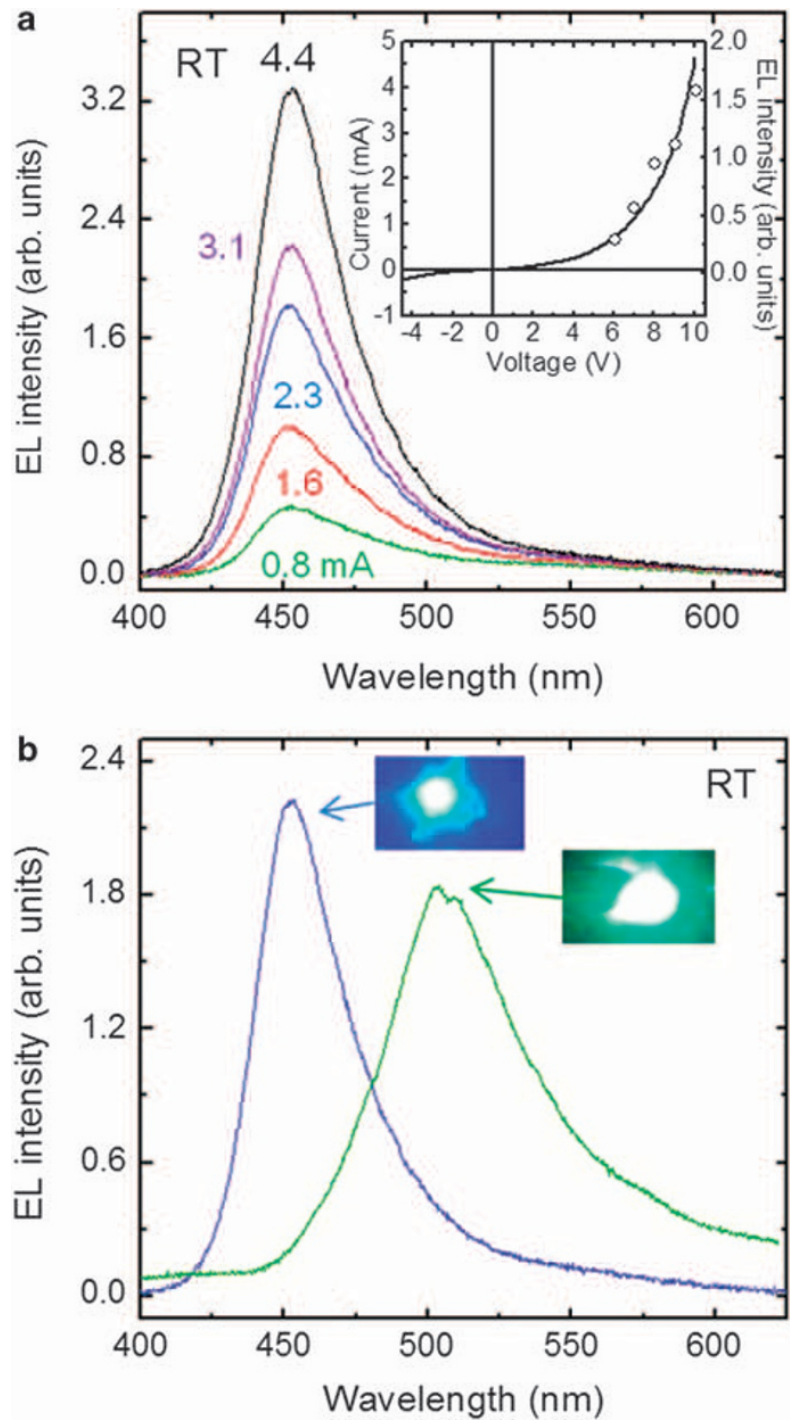

Figure 5 Characteristics of GaN-based thin-film LEDs fabricated on graphene-coated amorphous silica substrates. (a) Power-dependent EL spectra measured at room temperature. The inset of a shows the current and integrated EL intensity as a function of the applied bias voltage. (b) Room temperature EL spectra and corresponding EL images of the LEDs made with $\operatorname{In}_{x} \mathrm{Ga}_{1-x} \mathrm{~N}$ grown at 800 and $760{ }^{\circ} \mathrm{C}$.

large-scale forms that takes advantage of both inorganic semiconductors and graphene films. Moreover, creating hybrid heterostructures on various substrates, including metal foils, may allow inorganic LEDs to be fabricated in flexible large-scale displays.

We further investigated the EL characteristics of the LEDs fabricated on the CVD graphene-coated $\mathrm{SiO}_{2}$ substrates. Room temperature EL spectra and the $I-V$ characteristics of the LEDs were measured at various applied current levels (Figure 5a). The EL intensity gradually increased without a considerable EL peak shift as the applied current increased from 0.8 to $4.4 \mathrm{~mA}$. Moreover, above the turn-on voltage of $6 \mathrm{~V}$, both the current and the integrated EL intensity of the LEDs increased simultaneously upon increasing the applied bias voltage (inset of Figure 5a). This result indicates that the EL mechanism involves carrier transport through the $p-n$ junctions in the LEDs. Furthermore, the emission color of the LEDs could be controlled by changing the growth temperature of $\operatorname{In}_{x} \mathrm{Ga}_{1-x} \mathrm{~N}$ QW 
layers because the level of indium incorporated into the QW layers depended on the growth temperature. ${ }^{21}$ As shown in Figure 5b, blue $(453 \mathrm{~nm})$ and green $(504 \mathrm{~nm})$ EL images were obtained from the LEDs with the $\operatorname{In}_{x} \mathrm{Ga}_{1-x} \mathrm{~N}$ layer grown at $800^{\circ} \mathrm{C}$ and $760^{\circ} \mathrm{C}$, respectively.

In conclusion, high-quality GaN epitaxial films were heteroepitaxially grown on large-size CVD graphene films using a $\mathrm{ZnO}$ nanowall intermediate layer. The structural and optical characteristics of GaN thin films grown on CVD graphene films were comparable to GaN films grown on graphene layers mechanically exfoliated from graphite powder. Furthermore, the simple technique used to obtain high-quality inorganic semiconductor thin films even on amorphous substrates makes it possible to fabricate LEDs on large-area noncrystalline solid substrates and to provide and manufacture large and cost-effective optoelectronic and electronic devices.

\section{CONFLICT OF INTEREST}

The authors declare no conflict of interest.

\section{ACKNOWLEDGEMENTS}

This work was financially supported by the National Creative Research Initiative Project (grant no. R16-2004-004-01001-0) of the Korea Science and Engineering Foundations (KOSEF) and the Future-based Technology Development Program (Nano Fields, 2010-0029325) through the National Research Foundation of Korea (NRF) funded by the Ministry of Education, Science and Technology.

1 Chung, K., Lee, C. -H. \& Yi, G. -C. Transferable GaN layers grown on ZnO-coated graphene layers for optoelectronic devices. Science 330, 655-657 (2010).

2 Kobayashi, Y., Kumakura, K., Akasaka, T. \& Makimoto, T. Layered boron nitride as a release layer for mechanical transfer of GaN-based devices. Nature 484, 223-227 (2011).

3 Park, W. I., Lee, C. -H., Lee, J. M., Kim, N. J. \& Yi, G. -C. Inorganic nanostructures grown on graphene layers. Nanoscale 3, 3522-3533 (2011).

4 Sirringhaus, H., Tessler, N. \& Friend, R. H. Integrated optoelectronic devices based on conjugated polymers. Science 280, 1741-1744 (1998).

5 Yoon, J., Jo, S., Chun, I. S., Jung, I., Kim, H. -S., Meitl, M., Menard, E., Li, X., Coleman, J. J., Paik, U. \& Rogers, J. A. GaAs photovoltaics and optoelectronics using releasable multilayer epitaxial assemblies. Nature 465, 329-333 (2010).

6 Yang, J., Banerjee, A. \& Guha, S. Amorphous silicon based photovoltaics-from earth to the 'final frontier'. Sol. Energy Mater. Sol. Cells 78, 597-612 (2003).

7 Özgür, Ü., Alivov, Y. I., Liu, C., Teke, A., Reshchikov, M. A., Doğan, S., Avrutin, V., Cho, S. -J. \& Morkoç, H. A comprehensive review of $\mathrm{ZnO}$ materials and devices. J. Appl. Phys. 98, 041301 (2005).

8 Forrest, S. R. The path to ubiquitous and low-cost organic electronic appliances on plastic. Nature 428, 911-918 (2004).

9 Ponce, F. A. \& Bour, D. P. Nitride-based semiconductors for blue and green lightemitting devices. Nature 386, 351-359 (1997).

10 Nakamura, S. The roles of structural imperfections in InGaN-based blue light-emitting diodes and laser diodes. Science 281, 956-961 (1998).

11 Bour, D. P., Nickel, N. M., Van de Walle, C. G., Kneisslm, M. S., Krusor, B. S., Mei, P. \& Johnson, N. M. Polycrystalline nitride semiconductor light-emitting diodes fabricated on quartz substrates. Appl. Phys. Lett. 76, 2182-2184 (2000).

12 Choi, J. H., Zoulkarneev, A., Kim, S. I., Baik, C. W., Yang, M. H., Park, S. S., Suh, H. Kim, U. J., Son, H. B., Lee, J. S., Kim, M., Kim, J. M. \& Kim, K. Nearly singlecrystalline GaN light-emitting diodes on amorphous glass substrates. Nat. Photonics. 5, 763-769 (2011).

13 Bae, S., Kim, H., Lee, Y., Xu, X., Park, J. -S., Zheng, Y., Balakrishnan, J., Lei, T., Kim, H. R., Song, Y. I., Kim, Y. -J., Kim, K. S., Özyilmaz, B., Ahn, J. -H., Hong, B. H. \& lijima, S. Roll-to-roll production of 30-inch graphene films for transparent electrodes. Nat. Nanotechnol 5, 574-578 (2010).

14 Yadav, B. S., Singh, S., Ganguli, T., Kumar, R., Major, S. S. \& Srinivasa, R. S. Highly oriented GaN films grown on ZnO buffer layer over quartz substrates by reactive sputtering of GaAs target. Thin Solid Films 517, 488-493 (2008).

15 Kim, K., Lee, Z., Regan, W., Kisielowski, C., Crommie, M. F. \& Zettl, A. Grain boundary mapping in polycrystalline graphene. ACS Nano 5, 2142-2146 (2011).

16 An, J., Voelkl, E., Suk, J. W., Li, X., Magnuson, C. W., Fu, L., Tiemeijer, P., Bischoff, M., Freitag, B., Popova, E. \& Ruoff, R. S. Domain (grain) boundaries and evidence of 'twinlike' structures in chemically vapor deposited grown graphene. ACS Nano $\mathbf{5}$, 2433-2439 (2011).

17 Huang, P. Y., Ruiz-Vargas, C. S., van der Zande, A. M., Whitney, W. S., Levendorf, M. P., Kevek, J. W., Garg, S., Alden, J. S., Hustedt, C. J, Zhu, Y., Park, J., McEuen, P. L. \& Muller, D. A. Grains and grain boundaries in single-layer graphene atomic patchwork quilts. Nature 469, 389-393 (2011).

18 Yoo, H., Chung, K., Choi, Y. S., Kang, C. S., Oh, K. H., Kim, M. \& Yi, G. -C. Microstructures of GaN thin films grown on graphene layers. Adv. Mater. 24, 515-518 (2012).

19 Khan, M. A., Olson, D. T., Vanhove, J. M. \& Kuznia, J. N. Vertical-cavity, roomtemperature stimulated-emission from photopumped GaN films deposited over sapphire substrates using low-pressure metalorganic chemical vapor-deposition. Appl. Phys. Lett. 58, 1515-1517 (1991).

20 Yablonskii, G. P., Lutsenko, E. V., Pavlovskii, V. N., Zubialevich, V. Z., Gurskii, A. L., Kalisch, H., Szymakowskii, A., Jansen, R. A., Alam, A., Dikme, Y., Schineller, B. \& Heuken, M. Luminescence and stimulated emission from GaN on silicon substrates heterostructures. Phys. Status Solidi A 192, 54-59 (2002).

21 Nakamura, S. III-V nitride based light-emitting devices. Solid State Commun. 102, 237-248 (1997).
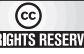

This work is licensed under the Creative Commons Attribution-NonCommercial-No Derivative Works 3.0 Unported License. To view a copy of this license, visit http:// creativecommons.org/licenses/by-nc-nd/3.0/

Supplementary Information accompanies the paper on the NPG Asia Materials website (http://www.nature.com/am) 